DOI:10.5392/IJoC.2011.7.1.008

\title{
An Energy Awareness Congestion Control Scheme in Wireless Sensor Networks
}

\author{
Mikyoung Kim, Junho Park, Dongook Seong, Dongwon Kwak \\ Department of Information and Communication Engineering \\ Chungbuk National University, Cheongju, Chungbuk, Korea. \\ Jaesoo Yoo \\ Department of Information and Communication Engineering \\ Chungbuk National University, Cheongju, Chungbuk, Korea.
}

\begin{abstract}
For energy-efficiency in Wireless Sensor Networks (WSNs), when a sensor node detects events, the sensing period for collecting the detailed information is likely to be short. The lifetime of WSNs decreases because communication modules are used excessively on a specific sensor node. To solve this problem, the TARP decentralized network packets to neighbor nodes. It considered the average data transmission rate as well as the data distribution. However, since the existing scheme did not consider the energy consumption of a node in WSNs, its network lifetime is reduced. In this paper, we propose an energy awareness congestion control scheme based on genetic algorithms in WSNs. The proposed scheme considers the remaining amount of energy and the transmission rate on a single node in fitness evaluation. Since the proposed scheme performs an efficient congestion control, it extends the network lifetime. In order to show the superiority of the proposed scheme, we compare it with the existing scheme through performance evaluation. It is shown that the proposed scheme enhances the data fairness and improves the network lifetime by about $27 \%$ on average over the existing scheme.
\end{abstract}

Keywords: Wireless Sensor Networks (WSNs), Genetic Algorithm, Congestion Control

\section{INTRODUCTION}

In recent, with the development of the computing technology and the semiconductor MEMS technology, the minimization, low cost, and low power of a sensor node used in wireless sensor networks have become possible. WSN acquires data of surrounding environment with sensors attached to each node. Therefore, WSN performs status monitoring, information transmission, and cooperative works with neighboring nodes in order to collect data that it is difficult for us to directly approach in various environments. For example, it is used in a variety of fields such as observation of wildlife's habitat, test of building's safety, military affair, environment, management of factory, monitoring of disaster, and medical service [1]. As the sensor nodes which are used in WSNs are mostly operated in the environments that we cannot access, it is impossible to replace the battery of each sensor node. For effective use of energy in the sensor nodes, it is necessary for each sensor node to reduce the number of transmissions in the network [2]. To

This is an excellent paper selected from the papers presented at ICCC 2010.

*Corresponding author.E-mail : yjs@chungbuk.ac.kr

Manuscript received Mar.01, 2011 ; accepted Mar.20, 2011 satisfy this requirement, when specific nodes detect the urgent event, sensor nodes increase the amount of data transmission. However, the short sensing cycle increases the data traffic of the sensor nodes in a routing path. Since the high data congested traffic causes a data queue overflow in the sensor nodes, sensor readings about urgent event can be lost [3]. To solve this problem, many researches on congestion control schemes are vigorously being progressed to alleviate the traffic congestion problems in WSNs.

Fig. 1 shows an example of the transmission of events detected by sensor nodes A, B, C, and D. When these sensor nodes detect an event, the sensor readings are transmitted to a sink node via the routing path shown by the lines. To collect more information about the event, these sensor nodes begin to increase their data sampling rates from 1packet/min to 100packets/min. The sensor readings concentrate on sensor node A with 300packets/min. As a result, sensor node A suffers from heavy traffic. This traffic load causes a queue overflow in sensor node A. The queue overflow results in the loss of important information about the urgent event. To avoid traffic congestion, the sensing data generated from nodes B, C, and D should be distributed to other neighboring parent sensor nodes except sensor node A. 


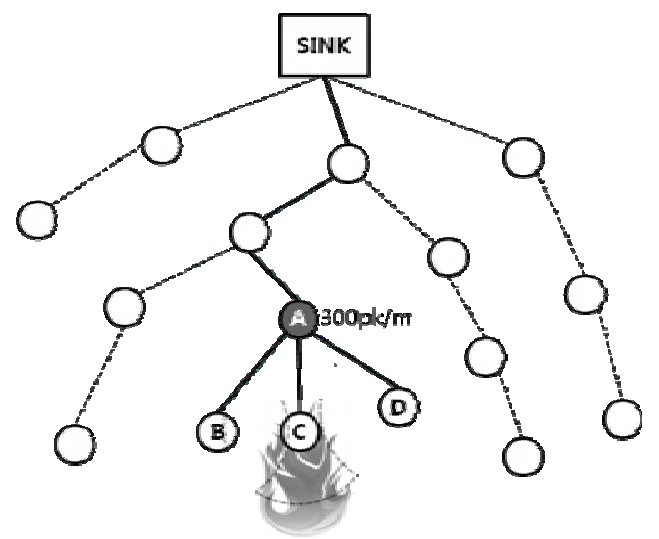

Fig.1. Event Detection and Traffic Congestion

In a method of solving traffic congestion problems, TARP [4], the sensor nodes distribute the traffic loads to their neighbor sensor nodes if it is concentrated on a specific node. At this moment, the sensor nodes perform a lightweight genetic algorithm based on the information about the child sensor nodes and their neighbor sensor nodes. Then, the sensor node selects available for forwarding the congested data and distributes the traffic loads evenly. However, since TARP considers only average data transmission rate and the data distribution, neighboring sensor nodes to a sink node along the path remain the high transmission rate.

To solve these problems, we proposes an novel energy awareness congestion control scheme based on genetic algorithms in wireless sensor networks considering additionally the remaining amount of energy and the transmission rate on a single node. The proposed scheme makes a detour a certain rates of the traffic to other nodes by performing genetic algorithms that based on information of the child nodes congested network traffic and the neighbor nodes of the child nodes. Because our scheme considers additionally the remaining amount of energy and the transmission rate, the fairness of data transmission of the entire network and network lifetime can be improved.

The rest of this paper is organized as follows. Section 2 reviews some problems of existing congestion controls and presents the related work on genetic algorithm. Section 3, we introduce our energy awareness congestion control scheme based on genetic algorithms. Section 4 shows simulated experiments and compares our results with the existing schemes. Finally, we conclude this paper and present some future works in Section 5.

\section{RELATED WORK}

\subsection{Congestion Control in WSNs}

Since the limited characteristic of network bandwidth, traffic congestion frequently occurs during the relay process of sensing data in wireless sensor networks. As a solution to this problem, several studies have been proposed the routing scheme to reduce of data sensing rates in terminal sensor nodes $[5,6]$.

The CODA (COngestion Detection and Avoidance in Sensor Network) proposed that the sensor nodes in the middle of the routing path monitor their input data traffic [5]. If the sensor node detects the data traffic more than a specific threshold, the result is transmitted to a sink node via multiple hops. In addition, a sink node monitors its own input data traffic to find traffic congestion. In short, when detecting traffic congestion, the sink node uses ACK messages to lower the data traffic congestion of the terminal sensor nodes detecting the events.

The ESRT (Event-to-Sink Reliable Transport Protocol) monitors their internal buffers to find traffic congestion to improve the reliability of data transmission in wireless sensor networks [6]. When traffic congestion is detected, a sink node collects event data, and broadcasts the congestion state to all sensor nodes. After, each sensor node tries to reduce the data transmission rate. As results, the traffic congestion problem could be alleviated gradually. When traffic congestion occurs, the previous methods required the reduction of data sensing rates in the terminal sensor nodes. In these methods, the sink node did not get enough information to the urgent events.

The TARP (Traffic-Aware Routing Protocol) detects the traffic congestion in each sensor node and uses the genetic algorithm to select data forwarding sensor nodes reducing data transfer rate of specific sensor node [4]. Due to the limited resources in wireless sensor nodes, this scheme uses a lightweight genetic algorithm that constraints the number of iteration loops to find a superior sensor node. However, the TARP considers the network traffic fairness by only using average of the transmission rate and the standard deviation. As a result, this scheme leads to other traffic congestion. As well as, the TARP exposes the greater energy consumption on the nodes located on the path to sink node. Accordingly, the neighbor sensor nodes toward the sink node along the path remain the high transmission rate. To resolve this problem, in this paper, we propose a new routing protocol considering amount of remaining energy and the data transmission rate of single node. The new routing scheme distributes the data traffic congestion of a specific sensor node.

\subsection{Genetic Algorithm (GA)}

The genetic algorithm is the search algorithm based on the principle of natural selection and the survival of the fittest [7]. This algorithm is a very wide range of applications because it has a simple structure and methods. Particularly, this algorithm has been widely used as engineering problem solution through an adaptive search and optimization. Fig.2 shows the flowchart of the genetic algorithm. First, an initial population of chromosomes is created to represent the solution to complex problems. Second, this algorithm performs the fitness evaluation of the original chromosomes and selects a parent chromosome to apply genetic operations. Third, the algorithm evolves the population through genetic operations such as crossover and mutation. Then, as the fitness function to newly created individual objects is applied, only the superior objects remain in the next generation. If a superior object does not exist, this algorithm repeats the loop iteration. 


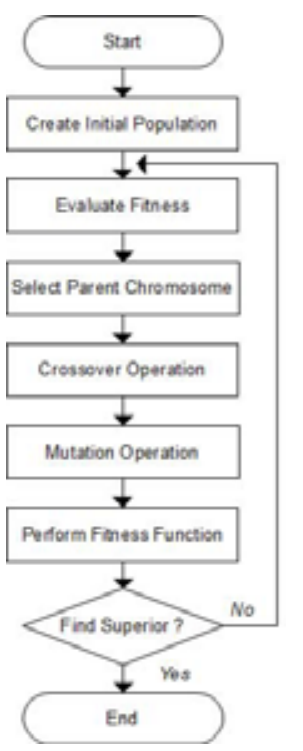

Fig.2. Genetic algorithm

To implement the genetic algorithm, chromosomes for the given problems are designed, and genetic operations are designated. In addition, a fitness function should be devised for target applications. The chromosomes could be composed as the binary denotation, permutation denotation or real integer representation.

\section{AN ENERGY AWARENESS CONGESTION CONTROL SCHEME BASED ON GENETIC ALGORITHMS IN WSNs}

The proposed scheme to perform the genetic algorithm periodically to gather the information of neighboring nodes, and the following types of chromosomes is produced.

\begin{tabular}{|c|c|c|c|c|c|c|}
\hline FIT & CID[n] & $\mathrm{CR}[\mathrm{n}]$ & $\mathrm{FR}[\mathrm{n}]$ & NID[2][n] & $\mathrm{NR}[2][\mathrm{n}]$ & NRE[2][n] \\
\hline & \multicolumn{6}{|c|}{ - FIT : FITness } \\
\hline & \multicolumn{6}{|c|}{ - CID : Child node ID } \\
\hline & \multicolumn{6}{|c|}{ - CR : Child node's data transfer Rate } \\
\hline & \multicolumn{6}{|c|}{ - FR : Forwarding Rate } \\
\hline & \multicolumn{6}{|c|}{ - NID : Neighbor node ID } \\
\hline & \multicolumn{6}{|c|}{ - NR : Neighbor node’s data transfer Rate } \\
\hline & \multicolumn{6}{|c|}{ - NRE : Neighbor node’s Residual Energy } \\
\hline
\end{tabular}

Fig.3. Representation of Chromosomes

The FIT is calculated via the entire fitness function, formula (4). Through the variation computation of fitness function, the new chromosomes are distributed to the child sensor nodes. Based on this information, the child sensor nodes distribute the congested traffic to their neighbor sensor nodes. A chromosome includes the basic information of the child node and their neighbor nodes of the child nodes. The CID and the CR include the number of the child node and the data transmission rate. The NID, NR and NRE include the information about neighbor nodes of the child node and the path information as well as the remaining amount of energy.
The FR represents a forwarding transmission rate of each child node, and it shows the data distribution for the other neighbor nodes. Then, the FR is newly assigned through a genetic algorithm whenever operations. By expressing this way, it is used as the basic information for evaluating the fitness by applying the genetic algorithm such as the crossover or mutation operation. Based on the chromosomes, the proposed scheme performs repeatedly the genetic algorithm, and decentralizes network traffic by disseminating of the genetic information to the child nodes.

Fig. 4 shows the routing topology of wireless sensor networks as an example. The sensor nodes 12,13 , and 14 are the children of sensor node 6. Fig. 4-(b) represents the chromosome structure of sensor node 6. As shown in the Fig. 4-(b), chromosomes of node 6 include the data forwarding rates allocated to the children, the data transfer rates of children sensor nodes, the data transfer rates of the neighboring sensor nodes of children sensor nodes and residual energy of neighbor nodes.

To select the data forwarding sensor node, the proposed scheme uses the data traffic rates of neighboring parent sensor nodes(upper level) within 2-hops of a child sensor node. In the Fig. 4-(a), sensor nodes 5 and 4 are the neighboring sensor nodes within 2-hops of child sensor node 12 . The sensor node 5 is within 1-hop of child sensor node 12, and sensor node 4 can be connected within 2-hops from sensor node 12 via sensor node 5. In the Figure 4-(b), we can see sensor nodes 5 and 4 as the NID[1], NID[2] items of this chromosome structure.

This chromosome structure has forwarding rates to each child sensor node as the FR items. The genetic algorithm allocates the FR values according to NRE in the formative step of the solution sets, and reallocates them through the mutation operations of the genetic algorithm.

The FIT represents the degree of fitness to current data forwarding rates. This field is allocated from executing the fitness function. Based on the FIT value of the chromosomes, the genetic algorithm can distinguish superior chromosomes from the solution sets.

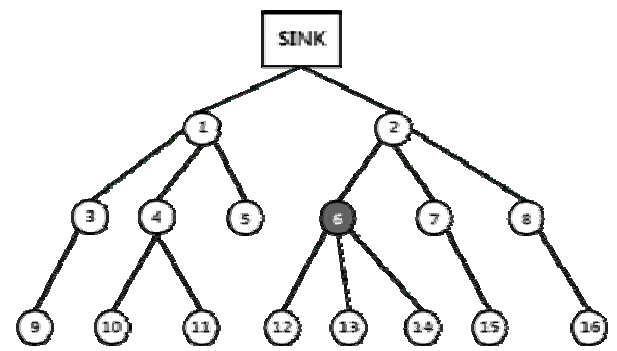

(a) Routing Topology of Wireless Sensor Networks

\begin{tabular}{|c|c|c|c|c|c|c|c|c|c|}
\hline FIT & (1) & CR & F & ND1 & Na & NFEI & $\mathrm{Fmaz}$ & MREZ & NAEZ \\
\hline \multirow{3}{*}{-} & 12 & 5 & $\bar{z}$ & 5 & 6 & $5 \pm$ & 4 & to & 67 \\
\hline & I3 & IO & 4 & 7 & 4 & 67 & a & $T$ & 39 \\
\hline & 14 & $T$ & 3 & 7 & 4 & 67 & 8 & 7 & 39 \\
\hline
\end{tabular}

(b) Chromosome Structure of Node 6.

Fig.4. Routing Topology and Chromosome Structure

The TARP places emphasis on the network traffic fairness by using average of the transmission rate and the standard 
deviation. But the proposed scheme distributes the traffic to each neighbor node. It decides optimal traffic distribution per each sensor node based on the sum of the fitness, formula (4) using the fitness function, formula (3). When the fitness function operates, furthermore, the proposed scheme processes the data dissemination to maximize the network lifetime by considering the remaining energy and the data transmission rate of each sensor node.

$$
\begin{gathered}
A D T=\sum_{i-n}^{n}\left(N R_{i}+F R\right) / n \\
D D T=\sqrt{\sum_{i-n}^{n}\left\{A D T-\left(N R_{i}+F R\right)\right\}^{2} / n} \\
\text { Fitness }(n)=\frac{\alpha \cdot N R E(n)}{D D T+\beta \cdot A D T+\gamma \cdot(N R(n)+F R(n))} \\
\text { EntireFitness }=\sum_{i=n}^{n}(\text { Fitness }(n))
\end{gathered}
$$

\section{PERFORMANCE EVALUATION}

\subsection{Simulation Setup}

To show the superiority of the proposed scheme, we have developed a simulator based on JAVA and have compared our scheme with the TARP. The performance evaluation was carried out through the simulation parameters shown in Table 1. We simulated a multi-hop network over the grid-based network topology. The model of the energy consumed for message transmission to a sensor node is \{MessageSize * $\left(\{\right.$ TransmissionCost $\}+\{$ AmplificationCost $\left.\} *\{\text { Distance }\}^{2}\right)$, where the transmission cost was $50 \mathrm{~nJ} / \mathrm{b}$ and the amplification cost was set as $100 \mathrm{pJ} / \mathrm{b} / \mathrm{m}^{2}$. The model of the energy consumed for receiving the message from a sensor node is \{MessageSize $*\{$ ReceiveCost $\}$, where the receiving cost was set as $50 \mathrm{~nJ} / \mathrm{b}$.

Table 1. Simulation Parameters

\begin{tabular}{cc}
\hline \hline Parameter & Default \\
\hline \hline Sensor ID size & 4byte \\
Sensor reading size & 4 byte \\
Topology & GRID \\
Location of sink node & $(0,0)$ \\
Location of event & $(100,100)$ \\
Initial Energy & $0.1 \mathrm{~J}$ \\
The \# of sensor nodes & 100 \\
\hline \hline
\end{tabular}

\subsection{Energy Consumption in the Network}

Fig. 5 shows the distribution of energy consumed by eventdriven data transmission in all sensor nodes. The TARP shows the greater energy consumption on the nodes located on the path to sink node $(0,0)$. The TARP reduces the energy consumption and the traffic congestion over the existing schemes. But it shows still relatively the high energy consumption of the node located on the path to sink node. As a result, this scheme has the disadvantage of decreasing network lifetime. On the other hand, the proposed scheme considers the remaining amount of energy and the transmission rate of the single node. In case similar conditions, we detour the congested data to neighbor nodes which have a lot of the remaining amount of energy. This figure shows the high fairness about the energy consumption on entire network by reducing the data transmission rate on the nodes located on the path to sink node.

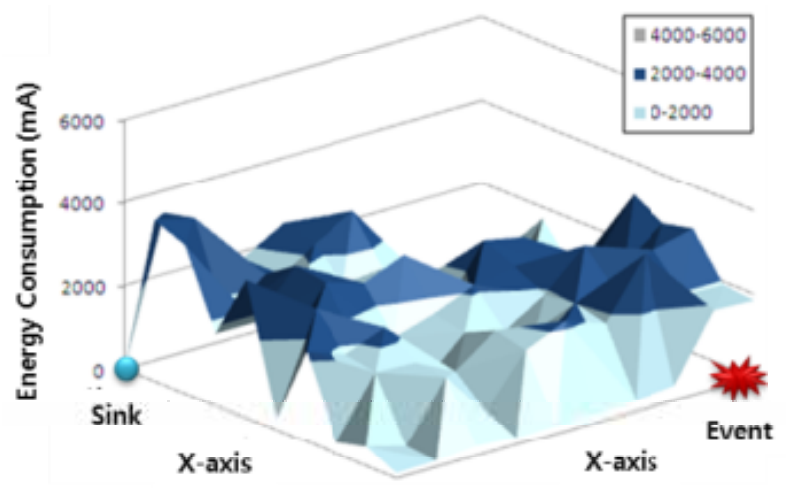

(a) TARP

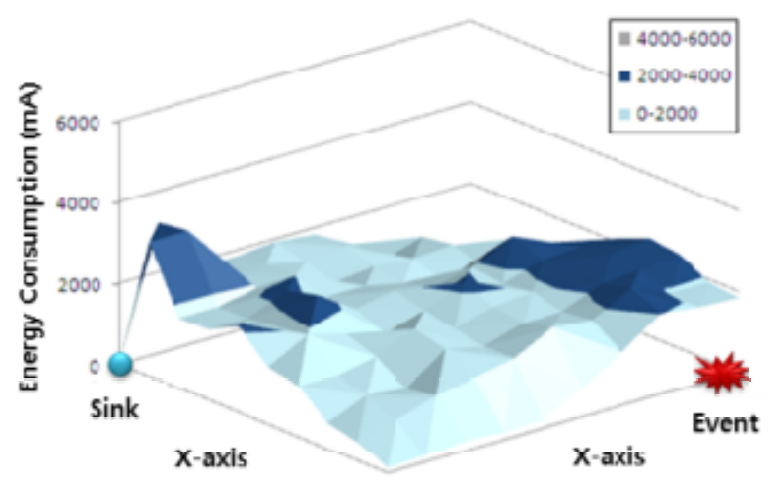

(b) Proposed Scheme

Fig.5. Energy Consumption per node

\subsection{Network Lifetime}

Fig. 6 shows the network lifetime according to the continual query processing. The MINT [8] has gained the low network lifetime because it leads to excessive energy consumption of the node located on the path to sink node by implementing the typical multi-hop routing. When the traffic congestion generates, the CODA [5] has gained higher the network lifetime because it declines the data transmission rate of the source node. The TARP is visible lower the network lifetime due to the large energy consumption of the sensor nodes located on the path to sink node. However, the proposed scheme increases the network lifetime by making a detour data to an unused sensor node considering the amount of the remaining energy of the sensor node. As a result, our scheme increases the network lifetime by $27 \%$ compared with the TARP. 


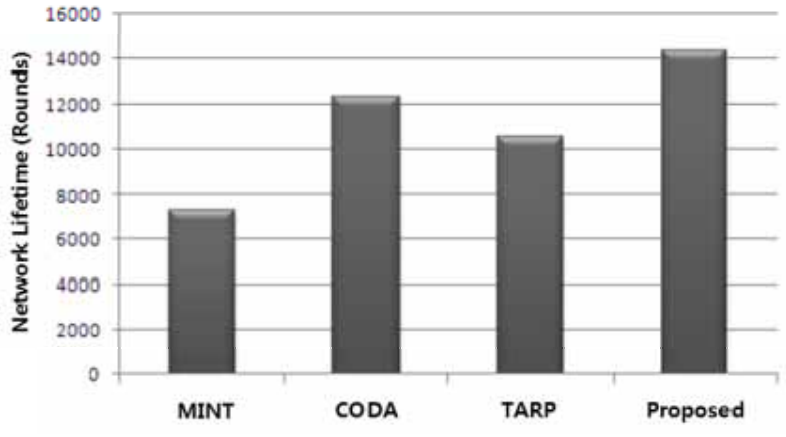

Fig.6. Network Lifetime

\section{CONCLUSIONS}

In this paper, we proposed an energy awareness congestion control scheme based on genetic algorithms. To do this, we analyzed the problems of the existing congestion control schemes. The proposed scheme considers the remaining amount of energy and the transmission rate on a single node in WSNs. Accordingly, our scheme maintains the high network lifetime over the existing scheme. Through various simulations, we compared the proposed scheme with the existing congestion control scheme, called TARP. As a result, our proposed scheme improves the network lifetime by about $27 \%$ and the fairness of data transmission on entire network over the existing scheme.

\section{ACKNOWLEDGEMENT}

This work was supported by the Ministry of Education, Science and Technology Grant funded by the Korea Government (The Regional Research Universities Program/Chungbuk BIT Research-Oriented University Consortium) and by the Ministry of Education, Science Technology (MEST) and Korea Industrial Technology Foundation (KOTEF) through the Human Resource Training Project for Regional Innovation.

\section{REFERENCES}

[1] Culler, D., Estrin, D., and Srivastava, M., "Guest Editors' Introduction: Overview of Sensor Networks,” IEEE Computer, vol.37, issue 8, pp.41-49, 2004.

[2] Cerpa, A., Elson, J., Estrin, D., Girod, L., Hamilton, M. and Zhao, J., "Habitat Monitoring: Application Driver for Wireless Communications Technology,” Proc. of ACM Workshop on Data Communications in Latin America and the Caribbean, pp.20-41, 2001.

[3] Wang, C., Li, B., Sohraby, K., Daneshmand, M. and $\mathrm{Hu}$, Y., "Upstream Congestion Control in Wireless Sensor Networks through Cross-Layer Optimization," IEEE Journal on Selected Areas in Communications, vol.25, pp.786-795, 2007.

[4] Park, C. and Jung, I., "Traffic-Aware Routing Protocol for Wireless Sensor Networks,” Proc. of International
Conference on Information Science and Applications, pp.1 8, 2010.

[5] Wan, C., Eisenman, S. and Campbell, A., "CODA : COngestion Detection and Avoidance in sensor networks," Proceedings of the 1st ACM Conference on Embedded Networked Sensor Systems, pp.266-279, 2003.

[6] Y. Sankarasubramaniam, O. B. Akan, and I. F. Akyildiz, "ESRT: Eventto-Sink Reliable Transport for Wireless Sensor Networks," Proceedings of the 4th ACM international symposium on Mobile ad hoc networking and computing, pp. 177-188, June 2003.

[7] D.E. Goldberg, "Genetic Algorithms in Search, Optimization and Machine Learning," AddisonWesley Publishing Company, Inc. 1989.

[8] Woo, A., Tong, T. and Culler, D., “Taming the Underlying Challenges of Reliable Multihop Routing in Sensor Networks," Proceedings of the 1st International Conference on Embedded Networked Sensor Systems, pp.14-27, 2003.

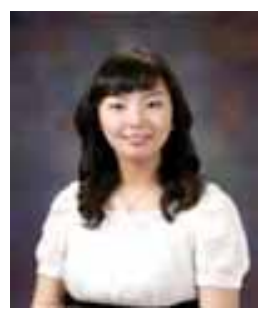

\section{Mikyoung Kim}

She received the B.S degree in Department of Information and Communication Engineering from Chungbuk National University, Korea in 2009. She is currently working towards M.S. degree on Department of Information and Communication Engineering. Hers main research interests include database system, wireless sensor network and bioinformatics.

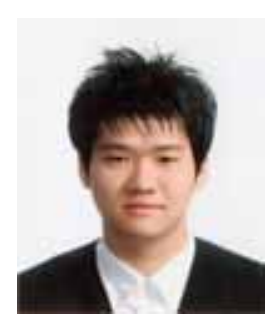

\section{Junho Park}

He received the B.S. and the M.S degree in the Departments of Information and Communication Engineering, Chungbuk National University, Korea in 2008 and 2010 respectively. He is working towards Ph.D degree on Department of Information and Communication Engineering from Chungbuk National University, Korea. His main research interests are the database system, wireless sensor network, RFID system, LMS, semantics and bioinformatics.

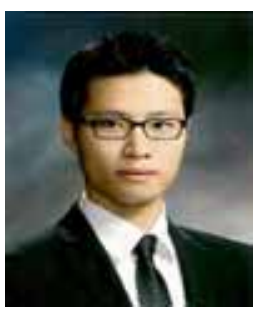

\section{Dongook Seong}

He received the B.S., M.S. and Ph.D. degrees in the department of Information and Communication Engineering from Chungbuk National University, South Korea in 2005, 2007 and 2011. He is now a post-Doc. in Korea Advanced institute of Science and Technology, Taejeon, South Korea. His main research interests are the database system, wireless sensor network and distributed storage system. 


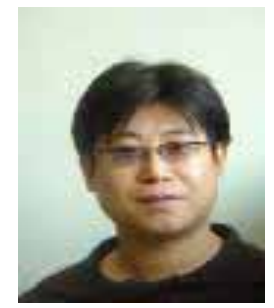

\section{Dongwon Kwak}

He received the B.S. and the M.S degree in the Departments of Information and Communication Engineering, Chungbuk National University, Korea in 2000 and 2002 respectively. He is working towards Ph.D degree on Department of Information and Communication Engineering from Chungbuk National University, Korea. His main research interests are the database system, distributed storage system and RFID system.

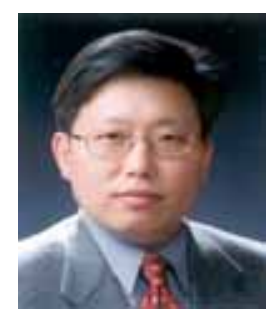

\section{Jaesoo Yoo}

He received the B.S. degree in Computer Engineering in 1989 from Chunbuk National University, Chunju, South Korea. And he received the M.S. and Ph.D. degrees in Computer Science in 1991 and 1995 from Korea Advanced Institute of Science and Technology, Taejeon, South Korea. He is now a professor in the department of Computer and Communication Engineering, Chungbuk National University, Cheongju, South Korea, where his research interests are the database system, multimedia database, 